\title{
Non-autonomous Dynamics of a Semi-Kolmogorov Population Model with Periodic Forcing
}

\author{
Tomás Caraballo ${ }^{\mathrm{a}}$, Renato Colucci ${ }^{\mathrm{b}}$, Xiaoying $\operatorname{Han}^{\mathrm{c}}$ \\ ${ }^{a}$ Departamento de Ecuaciones Diferenciales y Análisis Numérico, Universidad de Sevilla \\ Apdo. de Correos 1160, 41080 Sevilla, Spain \\ ${ }^{b}$ Department of Mathematical Sciences, Xi'an Jiaotong-Liverpool University \\ 111 Ren'ai Road, SIP, Suzhou P. R. China 215123 \\ ${ }^{c}$ Department of Mathematics and Statistics, 221 Parker Hall, Auburn University \\ Auburn, AL 36849, USA
}

\begin{abstract}
In this paper we study a semi-Kolmogorov type of population model, arising from a predator-prey system with indirect effects. In particular we are interested in investigating the population dynamics when the indirect effects are time dependent and periodic. We first prove the existence of a global pullback attractor. We then estimate the fractal dimension of the attractor, which is done for a subclass by using Leonov's theorem and constructing a proper Lyapunov function. To have more insights about the dynamical behavior of the system we also study the coexistence of the three species. Numerical examples are provided to illustrate all the theoretical results.
\end{abstract}

Key words: nonautonomous dynamical system, population dynamics, pullback attractor.

2000 MSC: Primary 92D25,Secondary 34C60.

\section{Introduction}

Indirect effect refers to species interactions which can occur through chains of direct species interaction, such as predation or interference competition. The studies of indirect effects are of great importance to biological sciences, as they can link the population dynamics of species that do not interact directly (see [3], [5], [7], [14], [27], [37], [39] and references therein). The following system represents a typical population model (see [11] and [12]) that describes indirect effects of predation for one predator (zooplankton, denoted by $Z$ ) and two preys

Email addresses: caraball@us.es (Tomás Caraballo), renatocolucci@hotmail.com (Renato Colucci), xzh0003@auburn.edu (Xiaoying Han) 
of different sizes (phytoplanjton, denoted by $C$ and $G$ ) :

$$
\left\{\begin{array}{l}
\dot{Z}=Z\left(-e+u_{c} C+u_{g} G\right), \\
\dot{C}=C\left[a_{c} I_{0}-\left(a_{c}+u_{c}\right) Z-a_{c} C-a_{c} G\right]-m_{1} C Z, \\
\dot{G}=G\left[a_{g} I_{0}-\left(a_{g}+u_{g}\right) Z-a_{g} C-a_{g} G\right]+m_{2} C Z .
\end{array}\right.
$$

The terms $-m_{1} C Z$ and $m_{2} C Z$ in system (1.1) describe the indirect effects generated by the fact that the predator prefers to predate the preys in the group with smaller size $(C)$ and the other group $(G)$ takes advantages of it. For the special case of $m_{1}=m_{2}=0$, any positive solution leads to the extinction of at least one of the three species (see [11]). For $m_{j}>0(j=1,2)$ uniform persistence has been shown, that there is a large variation of parameter sets with which any positive solution exhibits coexistence. In particular, sufficient conditions under which the attractor of the system is a fixed point or a stable limit cycle are found in [12] by using Hopf bifurcation.

Note that indirect effects can be of seasonal type (see for example [27], [34]), thus it is natural to consider a non-autonomous counterpart of system (1.1) where constants $m_{j}$ are replaced by time dependent terms $m_{j}(t)$. A simple choice of the time dependent terms is:

$$
m_{j}(t):=m_{j}|\sin (\omega t)|, \quad j=1,2 .
$$

The system is of particular interest when $m_{2}>m_{1}$. In fact, summing the second and third equation of (1.1) with $m_{j}(t)$ as in (1.2) gives

$$
\dot{C}+\dot{G}=\left(a_{c} C+a_{G} G\right)\left(I_{0}-Z-C-G\right)-Z\left(u_{c} C+u_{g} G\right)+\left(m_{2}-m_{1}\right)|\sin \omega t|,
$$

for which we expect a more complicated behavior of the solutions as the last term of (1.3) is nonnegative.

Numerical simulations provided in [12] showed that with non-autonomous perturbation the system can exhibit not only the periodic orbit but also period bifurcation phenomena. It has also been observed (see e.g. [34] for a two dimensional example or [4] for a periodically forced system) that if the autonomous system has a periodic behavior then the non-autonomous system may behave similarly to forced oscillators ([34]).

In this paper we consider a generalized formulation of system (1.1):

$$
\left\{\begin{array}{l}
\dot{x}(t)=x(t)\left(-b_{1}+a_{12} y(t)+a_{13} z(t)\right), \\
\dot{y}(t)=y(t)\left(b_{2}-a_{21} x(t)-a_{22} y(t)-a_{23} z(t)\right)-g_{1}(t) x(t) y(t), \\
\dot{z}(t)=z(t)\left(b_{3}-a_{31} x(t)-a_{32} y(t)-a_{33} z(t)\right)+g_{2}(t) x(t) y(t),
\end{array}\right.
$$

in which all the parameters $a_{i, j}, b_{k}(i, j, k=1,2,3)$ are positive.

Here we assume that $g_{1}(t)$ and $g_{2}(t)$ in the periodic forcing terms satisfy

$$
0 \leq g_{j}(t) \leq G_{j}, \quad \forall t \in \mathbb{R}, \quad j=1,2,
$$


and $G_{2}>G_{1}$. A possible choice of $g_{j}(t)$ arising from population models with indirect effects could be

$$
g_{j}(t)=G_{j}\left|\sin \left(\omega_{j} t+\phi_{j}\right)\right|,
$$

with $G_{2}>G_{1}>0$ and $\omega_{j}, \phi_{j}>0,(j=1,2)$. It is worth mentioning that it is possible to replace the $x y$ term in the forcing by a more general nonlinear form.

Observe that system (1.4) is not of the exact Kolmogorov type, as the third equation does not attain the Kolmogorov structure. Therefore this system can be regarded as a "semi-Kolmogorov-type" predator-prey system, which is different from traditional Kolmogorov systems, and is of more mathematical interests.

The rest of the paper is organized as follows. In Section 2 we provide some definitions and preliminary results from the theory of nonautonomous dynamical systems. In Section 3 we prove the existence of a pullback attractor for proper choice of the parameters. In Section 4 we estimate the Hausdorff dimension of the pullback attractor for a class of subsystems of (1.4). In Section 5 we study the coexistence of the three species, and a conclusion is given in Section 6 .

\section{Preliminaries}

In this section we provide some preliminaries from the general theory of non-autonomous dynamical systems [20] that we require in the sequel. As this theory is now well known and developed, we will only recall the necessary results for our study. The reader can find a nice introduction and motivation in the aforementioned paper [20] and the bibliography therein.

Consider an initial value problem for a non-autonomous ordinary differential equation in $\mathbb{R}^{d}$,

$$
\frac{d \mathbf{x}(t)}{d t}=F(t, \mathbf{x}), \quad \mathbf{x}\left(t_{0}\right)=\mathbf{x}_{0} .
$$

The solution usually depends on both the actual time $t$ and the initial time $t_{0}$ rather than just on the elapsed time $t-t_{0}$ as in an autonomous system. The solution mapping $\mathbf{x}\left(t, t_{0}, \mathbf{x}_{0}\right)$ of any initial value problem for which an existence and uniqueness theorem holds then satisfies (1) the initial value property that $\mathbf{x}\left(t_{0}, t_{0}, \mathbf{x}_{0}\right)=\mathbf{x}_{0},(2)$ the two-parameter semigroup evolution property that

$$
\mathbf{x}\left(t_{2}, t_{0}, \mathbf{x}_{0}\right)=\mathbf{x}\left(t_{2}, t_{1}, \mathbf{x}\left(t_{1}, t_{0}, \mathbf{x}_{0}\right)\right), \quad t_{0} \leq t_{1} \leq t_{2},
$$

and (3) the continuity property that $\left(t, t_{0}, \mathbf{x}_{0}\right) \mapsto \mathbf{x}\left(t, t_{0}, \mathbf{x}_{0}\right)$ is continuous on the state space $\mathbb{R}^{d}$. These properties of the solution mapping of non-autonomous ordinary differential equations motivates the process formulation of a non-autonomous dynamical system.

Definition 2.1. A process $\varphi$ on space $\mathbb{R}^{d}$ is a family of mappings

$$
\varphi\left(t, t_{0}, \cdot\right): \mathbb{R}^{d} \rightarrow \mathbb{R}^{d}, t \geq t_{0},
$$

which satisfies 
(i) initial value property: $\varphi\left(t_{0}, t_{0}, \mathbf{x}\right)=\mathbf{x}$ for all $\mathbf{x} \in \mathbb{R}^{d}$ and any $t_{0} \in \mathbb{R}$;

(ii) two-parameter semigroup property: for all $\mathbf{x} \in \mathbb{R}^{d}$ and $t_{2} \geq t_{1} \geq t_{0}$ it holds

$$
\varphi\left(t_{2}, t_{0}, \mathbf{x}\right)=\varphi\left(t_{2}, t_{1}, \varphi\left(t_{1}, t_{0}, \mathbf{x}\right)\right),
$$

(iii) continuity property: the mapping $\left(t, t_{0}, \mathbf{x}\right) \mapsto \varphi\left(t, t_{0}, \mathbf{x}\right)$ is continuous for any $\mathbf{x} \in \mathbb{R}^{d}$ and $t \geq t_{0}$.

Definition 2.2. Let $\varphi$ be a process on $\mathbb{R}^{d}$. A family $\mathcal{B}=\{B(t): t \in \mathbb{R}\}$ of nonempty subsets of $\mathbb{R}^{d}$ is said to be $\varphi$-invariant if $\varphi\left(t, t_{0}, B\left(t_{0}\right)\right)=B(t)$ for all $t \geq t_{0}$ and $\varphi$ - positively invariant if $\varphi\left(t, t_{0}, B\left(t_{0}\right)\right) \subseteq B(t)$ for all $t \geq t_{0}$.

Definition 2.3. Let $\varphi$ be a process on $\mathbb{R}^{d}$. A $\varphi$-invariant family $\mathcal{A}=\{A(t): t \in \mathbb{R}\}$ of nonempty compact subsets of $\mathbb{R}^{d}$ is called a forward attractor of $\varphi$ if it forward attracts all families $\mathcal{D}=\{D(t): t \in \mathbb{R}\}$ of nonempty bounded subsets of $\mathbb{R}^{d}$, i.e.,

$$
\operatorname{dist}\left(\varphi\left(t, t_{0}, D\left(t_{0}\right)\right), A(t)\right) \rightarrow 0 \quad \text { as } t \rightarrow \infty \quad\left(t_{0} \text { fixed }\right),
$$

and is called a pullback attractor of $\varphi$ if it pullback attracts all families $\mathcal{D}=$ $\{D(t): t \in \mathbb{R}\}$ of nonempty bounded subsets of $\mathbb{R}^{d}$, i.e.,

$$
\operatorname{dist}\left(\varphi\left(t, t_{0}, D\left(t_{0}\right)\right), A(t)\right) \rightarrow 0 \quad \text { as } t_{0} \rightarrow-\infty \quad(t \text { fixed }) .
$$

The existence of a pullback attractor follows from that of a pullback absorbing family, which is usually more easily determined.

Definition 2.4. A family $\mathcal{B}=\{B(t): t \in \mathbb{R}\}$ of nonempty compact subsets of $\mathbb{R}^{d}$ is called a pullback absorbing family for a process $\varphi$ if for each $t_{1} \in \mathbb{R}$ and every family $\mathcal{D}=\{D(t): t \in \mathbb{R}\}$ of nonempty bounded subsets of $\mathbb{R}^{d}$ there exists some $T=T\left(t_{1}, \mathcal{D}\right) \in \mathbb{R}^{+}$such that

$$
\varphi\left(t_{1}, t_{0}, D\left(t_{0}\right)\right) \subseteq B\left(t_{1}\right) \quad \text { for all } t_{0} \in \mathbb{R} \text { with } t_{0} \leq t_{1}-T .
$$

The proof of the following theorem is well known, see e.g., [20].

Theorem 2.5. Suppose that a process $\varphi$ on $\mathbb{R}^{d}$ has a $\varphi$-positively invariant pullback absorbing family $\mathcal{B}=\{B(t): t \in \mathbb{R}\}$ of nonempty compact subsets of $\mathbb{R}^{d}$.

Then $\varphi$ has a unique global pullback attractor $\mathcal{A}=\{A(t): t \in \mathbb{R}\}$ with its component sets determined by

$$
A(t)=\bigcap_{t_{0} \leq t} \varphi\left(t, t_{0}, B\left(t_{0}\right)\right) \quad \text { for each } t \in \mathbb{R} .
$$

If $\mathcal{B}$ is not $\varphi$-positively invariant, then

$$
A(t)=\bigcap_{s \geq 0} \overline{\bigcup_{t_{0} \leq t-s} \varphi\left(t, t_{0}, B\left(t_{0}\right)\right)} \text { for each } t \in \mathbb{R} .
$$




\section{Existence of pullback attractor}

In this section we will show the existence of a pullback attractor for system (1.4) in $\mathbb{R}^{3}$. First, thanks to the existence and uniqueness of solutions to (1.4), we can define a process $\left\{\varphi\left(t, t_{0}\right)\right\}_{t \geq t_{0}}$ in $\mathbb{R}^{3}$ by

$$
\varphi\left(t, t_{0}, \mathbf{x}_{0}\right)=\mathbf{x}\left(t ; t_{0}, \mathbf{x}_{0}\right), \quad \forall \mathbf{x}_{0} \in \mathbb{R}^{3} .
$$

Moreover, the process defined by (3.1) is continuous in $\mathbb{R}^{3}$.

Observe that the planes $x=0$ and $y=0$ are invariant in the sense that $\left.\dot{x}\right|_{x=0, y, z \geq 0}=0$ and $\left.\dot{y}\right|_{y=0, x, z \geq 0}=0$. Moreover $\left.\dot{z}\right|_{z=0, x, y \geq 0} \geq 0$. Hence by the continuity of solutions, any solution trajectory which starts from $\mathbb{R}_{+}^{3}=$ $\{(x, y, z): x \geq 0, y \geq 0, z \geq 0\}$ will stay there forever, which implies that $\mathbb{R}_{+}^{3}$ is positively invariant. Let

$$
N(t):=x(t)+y(t)+z(t),
$$

and sum the equations of system (1.4) to obtain

$$
\begin{aligned}
\dot{N}= & x\left(-b_{1}+a_{12} y+a_{13} z\right)+y\left(b_{2}-a_{21} x-a_{22} y-a_{23} z\right) \\
& -g_{1}(t) x y+z\left(b_{3}-a_{31} x-a_{32} y-a_{33} z\right)+g_{2}(t) x y .
\end{aligned}
$$

Next we study the sufficient conditions for the existence of global pullback attractors.

\subsection{Existence of pullback attractor}

For simplicity, denote

$$
\begin{aligned}
a & :=\min \left\{a_{21}-a_{12}, a_{22}, a_{23}, a_{31}-a_{13}, a_{32}, a_{33}\right\} \\
g & :=\sup _{t \in \mathbb{R}}\left[g_{2}(t)-g_{1}(t)\right], \quad b:=\max \left\{b_{2}, b_{3}\right\} .
\end{aligned}
$$

The following theorem provides a sufficient condition for the existence of a global attractor of system (1.4).

Theorem 3.1. System (1.4) possesses a global pullback attractor provided that

$$
a>g>0 \text {. }
$$

Proof. By (3.2) we have

$$
\begin{aligned}
\dot{N}= & -b_{1} x+b_{2} y+b_{3} z+\left[g_{2}(t)-g_{1}(t)\right] x y \\
& -\left[\left(a_{21}-a_{12}\right) x y+\left(a_{31}-a_{13}\right) x z+a_{22} y^{2}+a_{23} y z+a_{32} y z+a_{33} z^{2}\right] \\
\leq & -b_{1} x+b(y+z)+g \cdot x y-a(x+y+z)(y+z) \\
\leq & (y+z) \cdot[b-(a-g) N]
\end{aligned}
$$

Now, for any $\varepsilon \geq 0$, define the set $B_{\varepsilon}$ as

$$
B_{\varepsilon}:=\left\{(x, y, z) \in \mathbb{R}_{+}^{3}: \quad x+y+z \leq \frac{b}{a-g}+\varepsilon\right\} .
$$

We first prove that $B_{\varepsilon}$ is positively invariant for any $\varepsilon \geq 0$. 
(i) For any solution of (1.1) starting from a point inside $B_{0}$, since $\mathbb{R}_{+}^{3}$ is positively invariant and $\dot{N}(t) \leq 0$ on $x+y+z(=N)=\frac{b}{a-g}$, we have $b-(a-g) N(t) \geq 0$ for all $t \geq t_{0}$. This yields the positive invariance of $B_{0}$. In addition, it follows from inequality (3.4) that

$$
\dot{N} \leq N[b-(a-g) N] .
$$

Integrating the Bernoulli type inequality (3.5) easily yields

$$
N(t) \leq \frac{b N\left(t_{0}\right)}{(a-g) N\left(t_{0}\right)+\left[b-(a-g) N\left(t_{0}\right)\right] \cdot e^{-b\left(t-t_{0}\right)}},
$$

which implies that

$$
\lim _{t \rightarrow \infty} N(t) \leq \frac{b}{a-g} \quad \text { and } \quad \lim _{t_{0} \rightarrow-\infty} N(t) \leq \frac{b}{a-g} .
$$

(ii) For any solution starting from a point $(x, y, z)$ in the set $B_{\varepsilon} \backslash B_{0}$, Eq. (3.4) implies that $\dot{N}\left(t_{0}\right) \leq 0$ and thus the solution cannot escape from $B_{\varepsilon}$. Therefore $B_{\varepsilon}$ is positively invariant.

We next show that $B_{\varepsilon}$ is absorbing for any $\varepsilon>0$. In fact, given any $\varepsilon>0$, let us choose $\hat{\varepsilon}$ such that $0<\hat{\varepsilon}<\varepsilon$. Consider the solution of (1.4) starting at time $t_{0} \in \mathbb{R}$ from a point such that $N\left(t_{0}\right) \geq \frac{b}{a-g}+\varepsilon>\frac{b}{a-g}+\hat{\varepsilon}$. Provided that such a solution satisfies $N(t) \geq \frac{b}{a-g}+\hat{\varepsilon}$ for $t \geq t_{0}$, the time derivative of $M(t):=N(t)-\left(\frac{b}{a-g}+\hat{\varepsilon}\right)$ satisfies

$$
\begin{aligned}
\dot{M} & \leq-b_{1} x+(y+z) \cdot\left[b-(a-g)\left(\frac{b}{a-g}+\hat{\varepsilon}\right)\right] \\
& \leq-b_{1} x-(y+z)(a-g) \hat{\varepsilon} \\
& \leq-\min \left\{b_{1},(a-g) \hat{\varepsilon}\right\} \cdot N \\
& \leq-\min \left\{b_{1},(a-g) \hat{\varepsilon}\right\} \cdot M .
\end{aligned}
$$

This is a linear inequality and can be integrated to obtain

$$
M(t) \leq M\left(t_{0}\right) e^{-\min \left\{b_{1},(a-g) \hat{\varepsilon}\right\}\left(t-t_{0}\right)} .
$$

Hence there exists a time $T_{\varepsilon}$ such that $M(t) \leq \varepsilon-\hat{\varepsilon}$ for $t-t_{0} \geq T_{\varepsilon}$, which implies that $N(t) \leq \frac{b}{a-g}+\varepsilon$ for $t-t_{0} \geq T_{\varepsilon}$.

Therefore the set $B_{\varepsilon}$ is a compact absorbing set for any $\varepsilon>0$ and absorbs (both forwardly and the pullback) all bounded sets of $\mathbb{R}_{+}^{3}$. This ensures the existence of a global pullback attractor

$$
\mathcal{A}_{1}=\left\{A_{1}(t): \quad t \in \mathbb{R}\right\} \subset B_{\varepsilon} \text { for all } \varepsilon>0,
$$

and consequently $\mathcal{A}_{1} \subset B_{0}$. 
Note that the hypothesis $a>0$ is consistent with our biological model (1.1), while the hypothesis $a>g$ is not necessarily exact but to restrict the magnitude of the phase displacement of the periodic forcing terms. In fact we can loosen the previous hypothesis of $a>g$ and assume instead that

$$
a>\frac{b}{b_{1}} \cdot g,
$$

which allows larger values of $g$ for $b_{1}>b$. For the readers' convenience we rewrite the time derivative of $N(t)$ as

$$
\begin{aligned}
\dot{N} & \leq-b_{1} x+b(y+z)+g x y-a(x+y+z)(y+z) \\
& =b N-a N^{2}+x\left(a N+g y-b_{1}-b\right) \\
& \leq N(b-a N)-x\left[\left(b+b_{1}\right)-(a+g) N\right] .
\end{aligned}
$$

When $\left(b+b_{1}\right)-(a+g) N \geq 0$, we have

$$
\dot{N} \leq N(b-a N) \text {. }
$$

When $\left(b+b_{1}\right)-(a+g) N \leq 0$, noticing that assumption (3.6) implies that

$$
\frac{b+b_{1}}{a+g}>\frac{b}{a} \quad \text { and } \quad b-a N \leq b+b_{1}-(a+g) N, \quad \forall N \geq 0,
$$

we have

$$
\dot{N} \leq N(b-a N)-x(b-a N)=(y+z)(b-a N) .
$$

Therefore, by using similar arguments to the ones in the proof of Theorem 3.1 , it can be proved that for any $\varepsilon>0$, the set

$$
\Delta_{\varepsilon}:=\left\{(x, y, z) \in \mathbb{R}_{+}^{3}: \quad x+y+z \leq \frac{b}{a}+\varepsilon\right\}
$$

is positively invariant and attracting (both forwardly and pullback) all bounded set of $\mathbb{R}_{+}^{3}$, which implies the existence of a global pullback attractor

$$
\mathcal{A}_{2}=\left\{A_{2}(t): \quad t \in \mathbb{R}\right\} \subset \Delta_{0} .
$$

In the rest of the paper we refer to $\mathcal{A}_{i}=\{A(t): t \in \mathbb{R}\}, i=1,2$, as the pullback attractor for the process (3.1) depending on which hypothesis we are considering, either (3.3) or (3.6).

\subsection{Numerical illustration}

In this subsection we consider some numerical simulations in order to illustrate the results obtained in the previous subsection. 


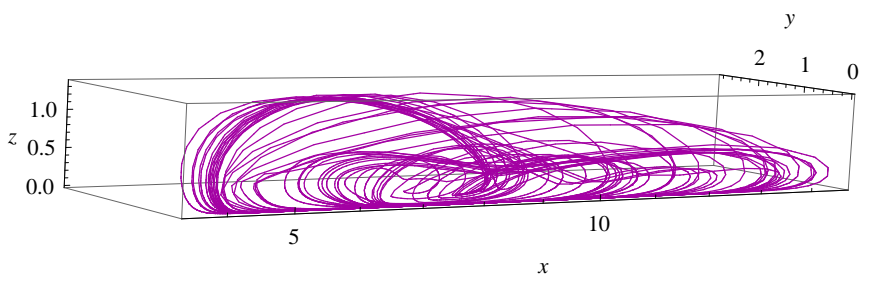

Figure 1: The Pullback Attractor $\mathcal{A}_{1}$.
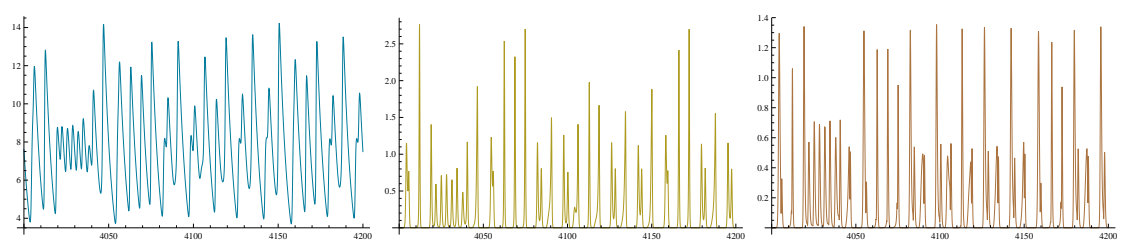

Figure 2: The time series of the functions $x, y$ and $z$.

Experiment 1. We consider system (1.4) with the following values of the parameters:

$$
\begin{aligned}
& a_{12}=0.75, a_{13}=0.25, a_{21}=0.8, a_{31}=0.8 \\
& a_{22}=a_{23}=a_{32}=a_{33}=b_{3}=1 \\
& b_{1}=0.2, b_{2}=10.6, g_{1}(t)=0.8|\sin t|, g_{2}(t)=1.4|\sin t|
\end{aligned}
$$

which satisfy the hypotheses (3.3) for the existence of a global pullback attractor $\mathcal{A}_{1}$ (see figure 1 for the attractor and figure 2 for $x, y, z$, as functions of time.).

From figures 1 and 2 we observe that the attractor is not trivial, and the non-periodicity of the functions $x(\cdot), y(\cdot), z(\cdot)$ suggests a chaotic behavior.

Experiment 2. In the second experiment we chose the values of the parameters in order to fulfill hypothesis (3.6) instead of (3.3) for the existence of a pullback attractor. Since larger values of $b_{1}$ are allowed, it is natural to expect that in this case the pullback attractor should contain some stable boundary fixed point leading to the extinction of at least one of the populations (the predator). This can be demonstrated by many numerical simulations. With the following values of the parameters we obtain the pullback attractor shown in 3 and $x, y, z$ as functions of time shown in 4).

$$
\begin{aligned}
& a_{12}=0.5, a_{13}=0.3, a_{21}=1, a_{31}=1.2, \\
& a_{22}=1.2, a_{23}=2, a_{32}=1.5, a_{33}=1.3, \\
& b_{1}=99, b_{2}=7, b_{3}=3 g_{1}(t)=|\sin t|, g_{2}(t)=8|\sin t|,
\end{aligned}
$$

Since extinction of one or more species appears under the condition (3.6), it is 


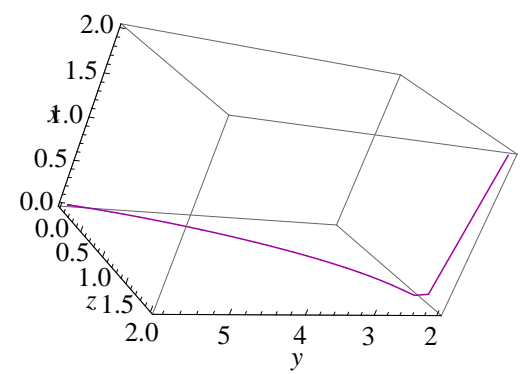

Figure 3: The Pullback Attractor $\mathcal{A}_{2}$ is trivial for both $g$ and $b_{1}$ large.

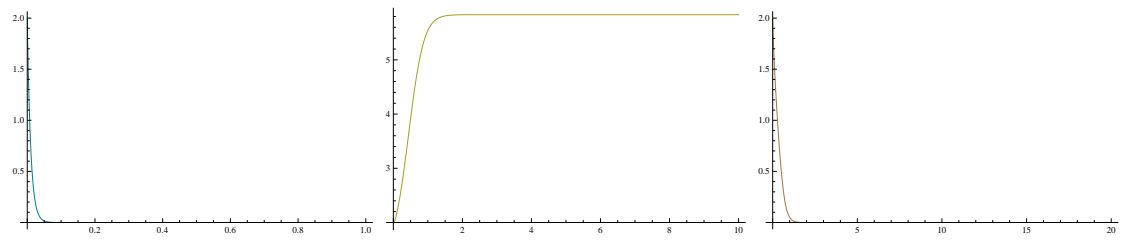

Figure 4: The time series of the functions $x, y, z$ on the attractor $\mathcal{A}_{2}$.

interesting to study whether it is possible to have a non-trivial pullback attractor in this case. In the next section we provide an estimate for the dimension of the pullback attractor.

\section{Dimension of the pullback attractor}

In order to have more insights about the structure of the pullback attractor, we next provide an upper estimate for the Hausdorff dimension of the pullback attractor. This will be done by using a method proposed by Leonov et al. in [24], which is based on the cocycle generated by nonautonomous differential equations.

To facilitate a better understanding, we write the nonautonomouns differential equations (1.4) in the form

$$
\dot{\mathbf{x}}=F(t, \mathbf{x}), \quad \mathbf{x}\left(t_{0}\right)=\mathbf{x}_{0} .
$$

Define the hull of $F$ with respect to equation (4.1) as

$$
\mathcal{H}(F):=\overline{\{F(\cdot+t, \cdot), t \in \mathbb{R}\}},
$$

where the closure is taken in the uniform convergence topology (see [10], [20] for more details). In fact, $\mathcal{H}(F)$ is a compact metric space if $F(t, \mathbf{x})$ is continuous in $\mathbf{x}$ and almost periodic in $t$. Define the base flow on $\mathcal{H}(F)$ by the shift mapping $\sigma_{t}: \mathcal{H}(F) \rightarrow \mathcal{H}(F)$

$$
\sigma_{t} \theta(\cdot, \cdot):=\theta(\cdot+t, \cdot), \quad t \in \mathbb{R}, \theta \in \mathcal{H}(F) .
$$


In Section 3 we have proved that the solutions mapping of system (1.4) defines a process (3.1) which possesses a pullback attractor $\mathcal{A}=\{A(t)\}_{t \in \mathbb{R}}$. To fit our system into the framework of cocycle dynamical systems, define the evaluation map $\tilde{F}: \mathcal{H}(F) \times \mathbb{R}^{3} \rightarrow \mathbb{R}^{3}$ via

$$
(\theta, \mathbf{x}) \in \mathcal{H}(F) \times \mathbb{R}^{3} \mapsto \tilde{F}(\theta, \mathbf{x})=\theta\left(t_{0}, \mathbf{x}\right) .
$$

Then equation (4.1) can be associated with

$$
\dot{\mathbf{x}}=\tilde{F}\left(\sigma_{t} \theta, \mathbf{x}\right)
$$

where $\theta \in \mathcal{H}(F)$ is arbitrary. Note that system (4.2) includes the original system (4.1) as a special case.

The cocycle generated by (4.2) is given by

$$
\psi(t, \theta) \mathbf{x}_{0}=\mathbf{x}\left(t ; \theta, \mathbf{x}_{0}\right),
$$

where $\mathbf{x}\left(t ; \theta, \mathbf{x}_{0}\right)$ denotes the solution to (4.2) with initial value $\mathbf{x}_{0}=\mathbf{x}(0)$. Taking $\theta=F \in \mathcal{H}(F)$ we have

$$
\psi(t, F) \mathbf{x}_{0}=\mathbf{x}\left(t ; F, \mathbf{x}_{0}\right),
$$

and hence (4.2) becomes

$$
\dot{\mathbf{x}}=\sigma_{t} F(0, \mathbf{x}), \quad \text { i.e. }, \quad \dot{\mathbf{x}}=F(t, \mathbf{x}) \text {. }
$$

Consequently we have

$$
\psi(t, F) \mathbf{x}_{0}=\varphi\left(t, t_{0}\right) \mathbf{x}_{0},
$$

which implies that system (1.4) generates a cocycle $\left(\{\psi(t, \theta)\}_{t \in \mathbb{R}, \theta \in \mathcal{H}(F)}, \mathbb{R}^{3}\right)$ over the base flow $\left(\left\{\sigma_{t}\right\}_{t \in \mathbb{R}}, \mathcal{H}(F)\right)$, with

$$
\psi\left(t, \sigma_{s} F\right) \mathbf{x}_{0}=\varphi(t+s, s) \mathbf{x}_{0} .
$$

For readers' convenience we state the main result in [24] as follows:

Theorem 4.1. Suppose that

(i) there exists a family of compact sets $\{\mathbb{A}(\theta)\}_{\theta \in \mathcal{H}(F)}$ which is negatively invariant for the cocycle defined by (4.3), i.e.

$$
\mathbb{A}\left(\sigma_{t} \theta\right) \subset \psi(t, \theta) \mathbb{A}(\theta), \quad \forall \theta \in \mathcal{H}(F), \quad t \in \mathbb{R} ;
$$

(ii) there exists a compact set $K \subset \mathbb{R}_{+}^{3}$ such that

$$
\overline{\bigcup_{\theta \in \mathcal{H}(F)} \mathbb{A}(\theta)} \subset K
$$


(iii) there exists a continuous function $V: \mathcal{H}(F) \times \mathbb{R}_{+}^{3} \rightarrow \mathbb{R}$ with derivatives $\left.\frac{d}{d t} V\left(\sigma_{t} \theta, \psi(t, \theta) \mathbf{x}_{0}\right)\right)$ along a given trajectory satisfying

$$
\begin{aligned}
\left.\frac{d}{d t} V\left(\sigma_{t} \theta, \psi(t, \theta) \mathbf{x}_{0}\right)\right)< & -\lambda_{1}\left(\sigma_{t} \theta, \psi(t, \theta) \mathbf{x}_{0}\right)-\lambda_{2}\left(\sigma_{t} \theta, \psi(t, \theta) \mathbf{x}_{0}\right) \\
& -s \lambda_{3}\left(\sigma_{t} \theta, \psi(t, \theta) \mathbf{x}_{0}\right)
\end{aligned}
$$

for all $t \in \mathbb{R}, \mathbf{x}_{0} \in K, \theta \in \mathcal{H}(F)$ and $s \in(0,1]$, where $\lambda_{i}, i=1,2,3$, are the eigenvalues of the symmetrized Jacobian of the right hand side (1.4) ordered as $\lambda_{1} \geq \lambda_{2} \geq \lambda_{3}$.

Then the Hausdorff dimension of the attractor for system (1.4) satisfies

$$
\operatorname{Dim}_{H}(\mathbb{A}(t)) \leq 2+s .
$$

This result has been widely used to study the Rossler and Lorenz systems (see [1], [2], [22], [23]). However it has not been applied to the Lotka-Volterra or Kolmogorov system due to their intrinsic complexity, such as the fully nonlinearity of the Lotka Volterra systems. Next we will verify the three conditions in Theorem 4.1 for system (1.4) to obtain an upper estimate of the Hausdorff dimension of the pullback attractor $\mathcal{A}=\{A(t)\}_{t \in \mathbb{R}}$ associated with the process defined in (3.1).

\subsection{Upper estimate of Hausdorff dimension of the pullback attractor}

First define the family $\{\mathbb{A}(\theta)\}_{\theta \in \mathcal{H}(F)}$ by

$$
\mathbb{A}(\theta)= \begin{cases}A(s), & \theta=\sigma_{s} F, \\ \left\{\mathbf{x} \in \mathbb{R}^{3}: \mathbf{x}=\lim _{t_{n} \rightarrow \infty} \mathbf{x}_{t_{n}}, \mathbf{x}_{t_{n}} \in A\left(t_{n}\right)\right\}, & \theta \neq \sigma_{s} F,\end{cases}
$$

where $s \in \mathbb{R}$ and $\theta \in \mathcal{H}(F)$. Then the set $\mathbb{A}(\theta)$ is compact for any $\theta \in \mathcal{H}(F)$. Morever the family $\{\mathbb{A}(\theta)\}_{\theta \in \mathcal{H}(F)}$ if negatively invariant. In fact, for $\theta=\sigma_{s} F$, by using (4.3) and the fact that $\{A(t)\}_{t \in \mathbb{R}}$ is invariant for process $\varphi$ defined by (3.1), we have $\psi(t, \theta) \mathbb{A}(\theta)=\psi\left(\sigma_{t} \theta\right)$ for all $t \geq t_{0}$. On the other hand for $\theta \neq \sigma_{s} F$, we have $\theta=\lim _{t_{n} \rightarrow \infty} \sigma_{t_{n}} F$ and it follows immediately that $\psi(t, \theta) \mathbb{A}(\theta) \supseteq \psi\left(\sigma_{t} \theta\right)$

Second, define the following compact set

$$
K:=\overline{\bigcup_{t \in \mathbb{R}} A(t)} \in \mathbb{R}^{3},
$$

we then have

$$
\overline{\bigcup_{\theta \in \mathcal{H}(F)} \mathbb{A}(\theta)} \subset K
$$

It then remains to show (4.4). Denote by $J$ the functional Jacobian of system (1.4), and let

$$
M:=\frac{J+J^{T}}{2}
$$


be the symmetrized Jacobian with $\operatorname{Tr}(M)=\operatorname{Tr}(J)$. Condition (4.4) can be rewritten by using the trace as

$$
\operatorname{Tr}(J)+(s-1) \lambda_{3}+\dot{V}<0,
$$

where $\lambda_{3}$ is the smallest eigenvalue of $M$.

To construct a proper Lyapunov type function,we start with a heuristic estimate of (4.6):

$$
\operatorname{Tr}(J)+u-\lambda_{3}+s\left(\lambda_{3}+v\right)<0,
$$

where

$$
u=\max _{\Delta_{0}}\{\dot{V}\}, \quad v=\max _{\Delta_{0}}\{-\dot{V}\} .
$$

and $\Delta_{0}$ is the smallest absorbing set containing the attractor.

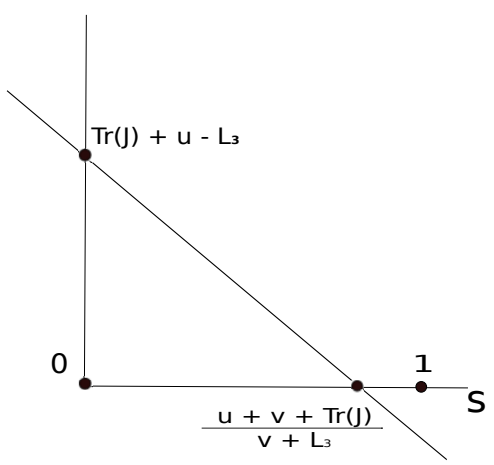

Figure 5: A graphical representation of inequality (4.7).

It can be seen in Fig. 5 that the inequality (4.7) represents a straight line. Hence in order to choose the minimum $s \in(0,1]$ satisfying (4.7), the following conditions need to be fulfilled inside $\Delta_{0}$ :

(a) the slope is negative, i.e.,

$$
\lambda_{3}+v<0
$$

(b) the intersection with the vertical axis is strictly positive, i.e.,

$$
\operatorname{Tr}(J)+u-\lambda_{3}>0
$$

(c) the intersection with the $s$-axis is in $(0,1)$, i.e.,

$$
u+v+\operatorname{Tr}(J)<0 .
$$

Conditions (a)-(c) above are rough but can provides useful indication for sharper estimates. For a better estimate we choose a Lyapunov function which depends explicitly on the eigenvalues $\mu_{i}$ of $J$, the Jacobian of the original system. To facilitate our analysis we assume that $V(\mathbf{x}, t)=V(\mathbf{x})$ and we can choose $V$ properly such that its total derivative satisfies

$$
\dot{V}=-\left(\mu_{1}+\mu_{2}+\mu_{3}\right)(1-s),
$$


where $\mu_{i}$ 's are the eigenvalues of $J$ with $\mu_{1} \geq \mu_{2} \geq \mu_{3}$. The inequality (4.4) then becomes:

$$
\lambda_{1}+\lambda_{2}+\lambda_{3}-\lambda_{3}+s \lambda_{3}-\left(\mu_{1}+\mu_{2}+\mu_{3}\right)(1-s)<0,
$$

which implies that

$$
s\left(\mu_{1}+\mu_{2}+\mu_{3}+\lambda_{3}\right)<\mu_{1}+\mu_{2}+\mu_{3}-\lambda_{1}-\lambda_{2}=\lambda_{3} .
$$

Now suppose (without loosing generality in the interesting cases) that:

$$
\operatorname{Tr}(M)=\operatorname{Tr}(J)<0,
$$

which implies that at least one eigenvalues of $M$ and $J$ is negative and as a consequence we have

$$
\lambda_{3}, \mu_{3}<0 .
$$

Hence

$$
s>\frac{\lambda_{3}}{\mu_{1}+\mu_{2}+\mu_{3}+\lambda_{3}}>0
$$

and the minimum value of $s$ that satisfies (4.1) is

$$
s^{*}=1-\frac{\mu_{1}+\mu_{2}+\mu_{3}}{\mu_{1}+\mu_{2}+\mu_{3}+\lambda_{3}}=1-\frac{\operatorname{Tr}(J)}{\operatorname{Tr}(J)+\lambda_{3}}=1-\frac{\operatorname{Tr}(A)}{\operatorname{Tr}(A)+\lambda_{3}} .
$$

It is clear that $s^{*} \in(0,1]$. Then by Leonov's theorem 4.1 we conclude that

$$
\operatorname{Dim}_{H}(A(t)) \leq 3-\frac{\operatorname{Tr}(J)}{\operatorname{Tr}(J)+\lambda_{3}},
$$

where $\lambda_{3}$ is the smallest eigenvalue of the symmetrized matrix $M$.

Remark 4.2. The above process can be generalized to a more general choice of $V$ satisfying

$$
\dot{V}=-\left(\mu_{1}+\mu_{2}+\mu_{3}\right)(1-s) \delta(x),
$$

where $\delta(x)>0$. By the same procedure of calculations we obtain

$$
s^{*}=1-\frac{\operatorname{Tr}(J)}{\operatorname{Tr}(J) \delta(x)+\lambda_{3}} .
$$

Note that (4.11) works provided that

$$
\frac{\operatorname{Tr}(J)}{\operatorname{Tr}(J) \delta(x)+\lambda_{3}}<1,
$$

which holds for any $\delta(x) \geq 1$

An estimate of the smallest eigenvalue $\lambda_{3}$ and bounds for the term $\frac{\operatorname{Tr}(J)}{\operatorname{Tr}(J) \delta(x)+\lambda_{3}}$ can be obtained by using the following result (see [38]): 
Theorem 4.3 (Wolkowicz-Styan). Let $M$ be a symmetric $n \times n$ matrix. Define the mean value $m$, and the variance $S$, of the eigenvalues of $M$ as

$$
m=\frac{\operatorname{Tr}(M)}{3}, \quad S^{2}=\frac{1}{n}\left[\sum_{k=1}^{n} \lambda_{k}^{2}-\frac{1}{n}\left(\sum_{k=1}^{n} \lambda_{k}\right)^{2}\right]=\frac{\operatorname{Tr}\left(M^{2}\right)}{n}-\frac{[\operatorname{Tr}(M)]^{2}}{n^{2}} .
$$

Then we have the following upper and lower bounds for the smallest eigenvalue $\lambda_{\min }(M)$ of $A$ :

$$
m-S(n-1)^{1 / 2} \leq \lambda_{\min }(M) \leq m-\frac{S}{(n-1)^{1 / 2}} .
$$

Note that the above estimates are to be performed on a suitable (the smallest) absorbing set containing the attractor of the system. In order to apply Leonov's theory to the Kolmogorov or Lotka-Volterra system we will need some modifications of the process described above. For readers' convenience, we restate the system (1.4) as follows

$$
\begin{aligned}
\dot{x} & =x f_{1}(y, z), \\
\dot{y} & =y f_{2}(x, y, z)-g_{1}(t) x y, \\
\dot{z} & =z f_{3}(x, y, z)+g_{2}(t) x y .
\end{aligned}
$$

where $f_{1}(y, z)=-b_{1}+a_{12} y+a_{13} z, f_{2}(x, y, z)=b_{2}-a_{21} x-a_{22} y-a_{23} z$, and $f_{3}(x, y, z)=b_{3}-a_{31} x-a_{32} y-a_{33} z$. The functional Jacobian is given by

$$
J=\left(\begin{array}{ccc}
f_{1} & x \frac{\partial f_{1}}{\partial y} & x \frac{\partial f_{1}}{\partial z} \\
y \frac{\partial f_{2}}{\partial x}-g_{1}(t) y & f_{2}+y \frac{\partial f_{2}}{\partial y}-g_{1}(t) x & y \frac{\partial f_{2}}{\partial z} \\
z \frac{\partial f_{3}}{\partial x}+g_{2}(t) y & z \frac{\partial f_{3}}{\partial y}+g_{2}(t) x & f_{3}+z \frac{\partial f_{3}}{\partial z}
\end{array}\right),
$$

where

$$
\frac{\partial f_{1}}{\partial x_{i}}>0, \quad \frac{\partial f_{i}}{\partial x_{j}}<0, \quad i=2,3, \quad j=1,2,3,
$$

and the trace has a simple expression of

$$
\operatorname{Tr}(J)=f_{1}+f_{2}+f_{3}+y \frac{\partial f_{2}}{\partial y}+z \frac{\partial f_{3}}{\partial z}-g_{1}(t) x .
$$

We next construct a Lyapunov type function depending on the trace $\operatorname{Tr}(J)$ in a simple way. To this end, we assume that the set

$$
\Delta_{0}:=\left\{(x, y, z) \in \mathbb{R}_{+}^{3}: \quad x+y+z \leq \beta\right\}, \quad \beta>0
$$

is an absorbing set. Then a possible choice of the Lyapunov function is

$$
V=k z-\ln \left(\frac{x}{\beta}\right)-\ln \left(\frac{y}{\beta}\right),
$$


where $k$ is a positive parameter to be determined later. This choice is motivated by the Kolmogorov type equations (4.12) and (4.13) of the non homogeneity of the equation (4.14).

Notice that $V(\mathbf{x}) \geq 0$ for $\mathbf{x} \in \Delta_{0}$. Moreover since $x=0$ and $y=0$ are invariant planes, $V$ is defined for solutions starting inside $\mathbb{R}_{+}^{3}$. The time derivative of (4.15) along the solution trajectories of the system (4.12) - (4.14) is:

$$
\begin{aligned}
& \dot{V}=k\left[z f_{3}+g_{2}(t) x y\right]-\left[f_{1}+f_{2}-g_{1}(t) x\right] \\
& =(k z+1) f_{3}+k g_{2}(t) x y+\left(y \frac{\partial f_{2}}{\partial y}+z \frac{\partial f_{3}}{\partial z}\right)-\operatorname{Tr}(J) \\
& :=\nu_{k}(x, y, z, t)-\operatorname{Tr}(J) .
\end{aligned}
$$

Substitute into the inequality (4.7) we have

$$
\operatorname{Tr}(J)+(s-1) \lambda_{3}+\nu_{k}(x, y, z, t)-\operatorname{Tr}(J)<0,
$$

which implies that

$$
s<1-\frac{\nu_{k}(x, y, z, t)}{\lambda_{3}} .
$$

For $s$ to satisfy $0<s \leq 1$, (4.16) essentially put a restrictions on $k$ as

$$
0 \leq \nu_{k}(x, y, z, t)<\lambda_{3}
$$

\subsection{Numerical Illustration}

In this subsection we provide an example by using the ideas of the previous subsection to estimate the dimension of the attractor for the system (1.4). In this case we use the following values of the parameters:

$$
\begin{aligned}
& a_{12}=\frac{3}{4}, a_{13}=\frac{1}{4}, a_{21}=0.8, a_{22}=1, a_{23}=1, \\
& a_{31}=0.8, a_{32}=1, a_{33}=1, b_{1}=0.2, b_{2}=8, b_{3}=0.41 \\
& g_{1}=0.8, g_{2}=1.4, w_{1}=w_{2}=1
\end{aligned}
$$

We use a more general Lyapunov type function:

$$
V(x, y, z)=k z-Q \ln \frac{x}{\beta}-P \ln \frac{y}{\beta},
$$

with $k=0, Q=-1$ and $P$ to be properly chosen later. Then:

$$
\dot{V}=f_{1}-P\left(f_{2}-g_{1}(t) x\right)
$$

and the inequality for the dimension satisfies

$$
\operatorname{Tr}(J)+(s-1) \lambda_{3}+\dot{V}<0
$$

To estimate the dimension numerically, we first use theorem (4.3) to estimate $\lambda_{3}$. Denote by $\hat{\lambda}_{3}$ the lower bound of $\lambda_{3}$ on $\Delta_{0}$, then it ought to satisfy

$$
\operatorname{Tr}(J)+(s-1) \hat{\lambda}_{3}+\dot{V}<0
$$


for any $t \geq 0$ on $\Delta_{0}$. Numerical simulations have shown that for $P=1.2$ and $s=0.4$ the inequality (4.17) holds, while for $s<0.4$ the inequality (4.17) can not be verified as the left hand side can take positive values. Hence an estimate of $s \leq 0.4$ can be obtained by calculating the minimum $s \in(0,1]$ to satisfy (4.17), and therefore

$$
\operatorname{Dim}(\mathcal{A})<2.4
$$

Note that this estimate can be improved by globally optimizing the choice of $P$ and $s$, yet here we demonstrate that the Leonov's method can be applied.

The following figures 6 and 7 represent the pullback attractor and the time series for $x, y$ and $z$, receptively.

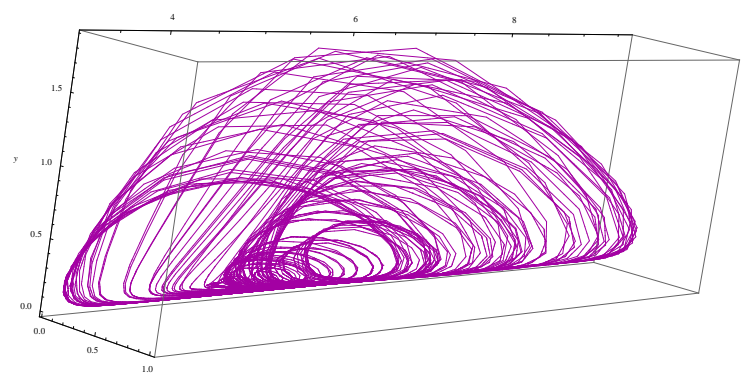

Figure 6: The Pullback Attractor $\mathcal{A}_{1}$ for the choice of the parameters as in subsection 4.2.

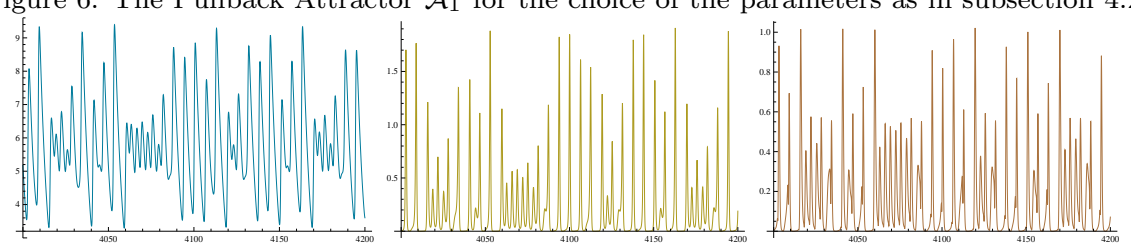

Figure 7: The time series of the functions $x, y, z$ on the attractor $\mathcal{A}_{1}$ for the choice of the parameters as in section 4.2 .

\section{Coexistence of the three species}

In this section we discuss the coexistence of the three competing species. However we remark that no general information is available about uniform persistence of the system (see [33]), which is one of the most useful properties from ecological and biological points of view. In this section we will consider the case of hypothesis (3.3).

For simplicity, set

$$
\begin{aligned}
\tilde{a} & :=\max \left\{a_{21}-a_{12}, a_{22}, a_{23}, a_{31}-a_{13}, a_{32}, a_{33}\right\}, \\
\tilde{b} & :=\min \left\{b_{2}, b_{3}\right\}, \quad \tilde{g}:=\inf _{t \in \mathbb{R}}\left\{g_{2}(t)-g_{1}(t)\right\} .
\end{aligned}
$$


Before discussing the coexistence of the species, we first construct a positive invariant subset of $\mathbb{R}_{+}^{3}$ in the next proposition.

Proposition 5.1. System (1.4) possesses a positive invariant set away from the origin, provided that

$$
\frac{\tilde{b}}{\gamma} \geq \frac{b_{1}}{a}
$$

Proof. By (1.4) we have

$$
\begin{aligned}
\dot{N}= & -b_{1} x+b_{2} y+b_{3} z+\left[g_{2}(t)-g_{1}(t)\right] x y \\
& -\left[\left(a_{21}-a_{12}\right) x y+\left(a_{31}-a_{13}\right) x z+a_{22} y^{2}+a_{23} y z+a_{32} y z+a_{33} z^{2}\right] \\
\geq & -b_{1} x+\tilde{b}(y+z)-\tilde{a}(y+z) N+\tilde{g} x y .
\end{aligned}
$$

When $\tilde{g} \geq 0$, from (5.2) we obtain

$$
\begin{aligned}
\dot{N} & \geq-b_{1} N+\tilde{b}(y+z)+\tilde{a}(y+z) N-2 \tilde{a}(y+z) N \\
& =N\left[\tilde{a}(y+z)-b_{1}\right]+(y+z)(\tilde{b}-2 \tilde{a} N) .
\end{aligned}
$$

While for $\tilde{g} \leq 0$, it also follows from (5.2) that

$$
\begin{aligned}
\dot{N} & \geq-b_{1} N+\tilde{b}(y+z)-\tilde{a}(y+z) N+\tilde{g}(y+z) N \\
& \geq-b_{1} N+\tilde{b}(y+z)+\tilde{a}(y+z) N-(2 \tilde{a}-\tilde{g})(y+z) N \\
& =N\left[\tilde{a}(y+z)-b_{1}\right]+(y+z)[\tilde{b}-(2 \tilde{a}-\tilde{g}) N] .
\end{aligned}
$$

Thus in summary we have

$$
\dot{N} \geq N\left[\tilde{a}(y+z)-b_{1}\right]+(y+z)(\tilde{b}-\gamma N), \quad \gamma:=\max \{2 \tilde{a}, 2 \tilde{a}-\tilde{g}\} .
$$

Define the set $\tilde{\Delta} \in \mathbb{R}_{+}^{3}$ to be

$$
\tilde{\Delta}:=\left\{(x, y, z) \in \mathbb{R}_{+}^{3}: \quad y+z \geq \frac{\tilde{b}}{\gamma}, \quad x+y+z \leq \frac{b}{a-g}\right\},
$$

then $\tilde{\Delta}$ is non-empty, since $\frac{\tilde{b}}{\gamma}<\frac{b}{a-g}$. Moreover since $\frac{\tilde{b}}{\gamma} \geq \frac{b_{1}}{a}$, any points in $\tilde{\Delta}$ satisfy $y+z \geq \frac{\tilde{b}}{\gamma}$, and consequently

$$
\dot{N} \geq \frac{b_{1}}{\tilde{a}}(\tilde{b}-\gamma N)
$$

Inequalities (5.5) and (3.5) together give the positive invariance of $\tilde{\Delta}$.

\subsection{Coexistence analysis}

Notice that condition (5.1) implies the smallness of $b_{1}$. In addition for $\tilde{\Delta}$ defined in (5.4) to be non-empty, $x$ needs to be less than the difference between $\frac{\tilde{b}}{\gamma}$ and $\frac{b}{a-g}$. These suggest the importance of the predator $(x)$ in order to avoid 
the extinction of species (or collapse) of the system. Next we discuss further the conditions under which none of the species will extinct. These will be done by constructing the conditions step by step.

(i) To avoid the extinction of $(x)$. In fact, for any solution starting from $\left(x_{0}, y_{0}, z_{0}\right) \in \tilde{\Delta}$, from the first equation of (1.4) we have

$$
\dot{x} \geq x\left(\min \left\{a_{12}, a_{13}\right\} \cdot \frac{\tilde{b}}{\gamma}-b_{1}\right)
$$

Thus if it holds that

$$
\min \left\{a_{12}, a_{13}\right\} \frac{\tilde{b}}{\gamma}>b_{1},
$$

the predator $(x)$ never goes to extinction.

(ii) To avoid the extinction of both $(y)$ and $(z)$. In fact, assume on the contrary $y=z=0$, system (1.4) is reduced to

$$
\dot{x}=-b_{1} x,
$$

which implies also the extinction of $(x)$. Therefore, assuming conditions (5.1) and (5.6), the predator $(x)$ and one of the prey $(y)$ or $(z)$ must persist.

(iii) To avoid the extinction of $(y)$, by showing that no invariant subsets of the plane $y=0$ can attract positive solutions. In fact, On the plane $y=0$ the system (1.4) becomes

$$
\left\{\begin{array}{l}
\dot{x}=x\left(a_{13} z-b_{1}\right), \\
\dot{z}=z\left(b_{3}-a_{31} x-a_{33} z\right),
\end{array}\right.
$$

which admits the following fixed points

$$
P=(0,0,0), \quad P_{y}=\left(\frac{a_{13} b_{3}-a_{33} b_{1}}{a_{13} a_{31}}, 0, \frac{b 1}{a_{13}}\right), \quad P_{1}=\left(0,0, \frac{b_{3}}{a_{33}}\right) .
$$

Since with hypotheses (5.1) and (5.6), $(x)$ and at least one of $(y)$ and $(z)$ must survive, then neither $P$ nor $P_{1}$ can attract positive solutions. On the other side, if $P_{y}$ does not exist then no periodic solutions exist on $y=0$ (see [11]) and the invariant sets of $y=0$ are just the fixed points $P$ and $P_{1}$. Thus a sufficient condition to avoid extinction of $(y)$ is (non existence of $P_{y}$ ) is

$$
a_{13} b_{3}-a_{33} b_{1}<0 \text {. }
$$

However condition (5.7) is not necessary, as it would be sufficient to show that $P_{y}$ is unstable directly for the original three dimensional system (1.4). So far we have that $(x)$ and $(y)$ will survive under hypotheses (5.1) and (5.6).

(iv) To avoid the extinction of $(z)$. First the plane $z=0$ is not invariant and. In addition, when $x, y \neq 0, z=0$ gives

$$
\dot{z}=g_{2}(t) x y \geq 0,
$$


which becomes zero if and only if $g_{2}(t)=0$. As a consequence there are no invariant sets in the plan $z=0$.

It is clear that $\left(0, \frac{b_{2}}{a_{22}}, 0\right) \in \tilde{\Delta}$ is an equilibrium on the $y$-axis. But this cannot attract positive solutions as its $x$ component should converge to zero and this is not possible under hypotheses (5.1) and (5.6). Then no positive solutions converge to the boundary of $\mathbb{R}_{+}^{3}$.

(v) To avoid heteroclinic cycles between the invariant sets of $\partial \mathbb{R}_{+}^{3}$ (see [21]), because it has been observed (see [33]) that such cycles can bring the extinction of some of the species in real situations subjected to deterministic or random perturbation. To this end, we check the connections between all the invariant sets of the boundary of $\mathbb{R}_{+}^{3}$. Since we have already analyzed the plane $y=0$ and $z=0$, it remains to investigate the invariant sets on the plane $x=0$, where (1.4) become

$$
\left\{\begin{array}{l}
\dot{y}=y\left(b_{2}-a_{22} y-a_{23} z\right), \\
\dot{z}=z\left(b_{3}-a_{32} y-a_{33} z\right),
\end{array}\right.
$$

Equations (5.8) possess the following fixed points

$$
\begin{gathered}
P=(0,0,0), \quad P_{1}=\left(0,0, \frac{b_{3}}{a_{33}}\right), \quad P_{2}=\left(0, \frac{b_{2}}{a_{22}}, 0\right), \\
P_{3}=\left(0, \frac{b_{2} a_{33}-b_{3} a_{23}}{a_{22} a_{33}-a_{23} a_{32}}, \frac{b_{3} a_{22}-b_{2} a_{32}}{a_{22} a_{33}-a_{23} a_{32}}\right),
\end{gathered}
$$

and have no periodic orbits (see [11] and references cited therein).

To facilitate the rest of the analysis we consider a sufficient condition for which $P_{3}$ falls outside of $\mathbb{R}_{+}^{3}$, i.e., one of the following inequalities is satisfied:

$$
b_{2} a_{33}-b_{3} a_{23}<0, \quad b_{3} a_{22}-b_{2} a_{32}<0 .
$$

Then together with hypotheses (5.1) and (5.6) we obtain that the invariant sets contained in the boundary of $\mathbb{R}_{+}^{3}$ are the fixed points $P, P_{1}$ and $P_{2}$, which are all contained in the invariant set $x=0$. They cannot be approached since they need the extinction of at least two species, which is not possible according to part (ii).

(vi) To avoid heteroclinic connections among the fixed points $P, P_{1}$ and $P_{2}$. In fact, the fixed point $P_{1}$ is on the $z$-axis which is stable, while the fixed point $P_{2}$ is on the $y$-axis which is also stable. Moreover inside the plane $x=0$, depending on choice of the parameters, all solutions converge to either $P_{1}$ or $P_{2}$. Thus there are no heteroclinic cycles, indeed the unique available connection is

$$
P \rightarrow P_{1}, \quad P \rightarrow P_{2}
$$

In summary we have the following result.

Theorem 5.2. Assume that the hypotheses (5.1), (5.6), and (5.9) are satisfied. Then the three species coexist. 


\subsection{Numerical illustrations}

The following numerical simulations are presented to illustrate the results of the present subsection.

Experiment 3. We consider a case that (5.1), (5.6), (5.7), and (5.9) are all fulfilled. We choose the parameters to be:

$$
\begin{aligned}
& a_{12}=1, a_{13}=1, a_{21}=1.5, \\
& a_{22}=1, a_{23}=1, a_{31}=1.5, a_{32}=1, a_{33}=1 \\
& b_{1}=0.4, b_{2}=1.5, b_{3}=1 g_{1}(t)=|\sin t|, g_{2}(t)=1.2|\sin t|,
\end{aligned}
$$

And we obtain that the attractor $\mathcal{A}_{1}$ is a small limit cycle. In this case we have coexistence of the three populations that is the existence of a small limit cycle (see figure 8 below).

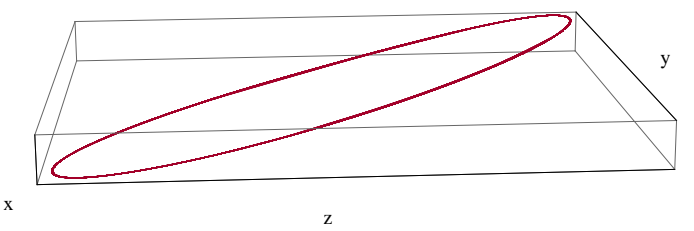

Figure 8: The Pullback Attractor $\mathcal{A}_{1}$ is a smal limt cycle.

Experiment 4. In this case we change the values of $b_{1}$ to 1 and keep all other parameters as in Experiment 3, then condition (5.7) is not satisfied. We still have coexistence (see figure 9 below) since (5.7) is not a necessary condition.

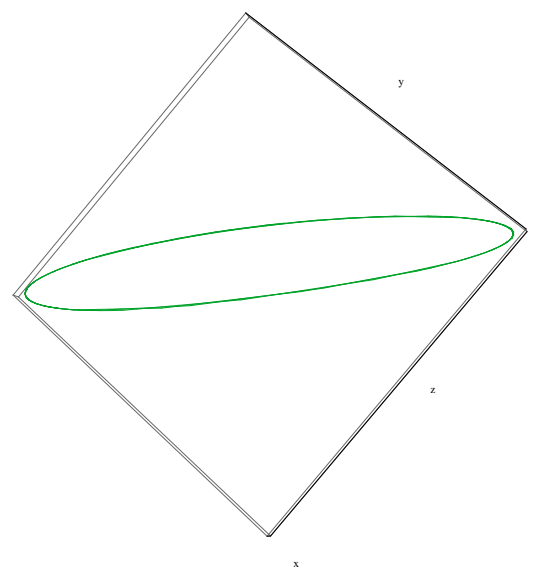

Figure 9: The Pullback Attractor $\mathcal{A}_{1}$ is again a smal limit cycle.

However if we further increase the magnitude of $b_{1}$, for example $b_{1}=1.5$. Then only populations $y$ and $z$ survive (see figure 10 below). 


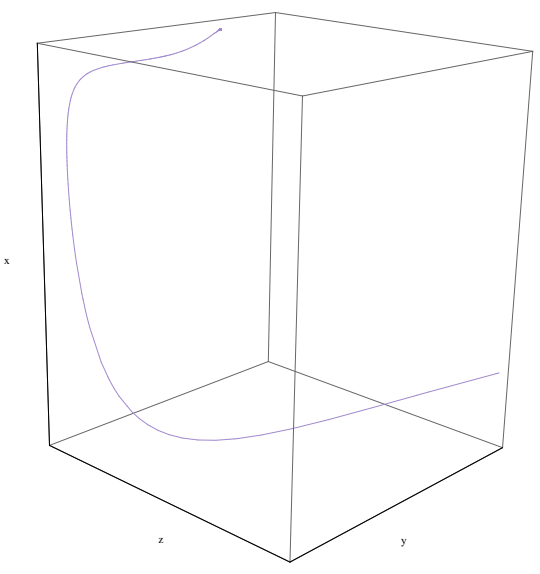

Figure 10: The Pullback Attractor $\mathcal{A}_{1}$ is a fixed point.

\section{Conclusions and final remarks}

In this article we have shown the existence of pullback attractors for a semiKolmogorov system (1.4). In addition, by using numerical experiments we have found scenarios in which the system show a chaotic behavior, and provided an upper bound for the Hausdorff dimension of the pullback attractor for these scenarios, by using Leonov theory. We have also discussed the coexistence of solutions, by constructing the hypotheses for which the invariant sets of the boundary of $\mathbb{R}_{+}^{3}$ are reduced to three fixed points (which cannot be connected by an heteroclinic cycle) and do not attract solutions. We note that the general problem of the uniform persistence of the system requires further study.

A possible way to obtain more insights of the coexistence is to consider the stability of boundary fixed points in the context of non autonomous stability theory (see [28]). In particular the following theorem of Cetaev (see [28]) can be used to study the instability of the origin $P$ :

Theorem 6.1. If for any disk $D$ of the phase space centered at the origin there exists a function $V$ and an open subset $\Theta \subset D$, such that, for all $t \geq t_{0}$

(i) $V$ is bounded in $\Theta$ and assumes positive values in $\Theta \cap \operatorname{int}(D)$,

(ii) $V$ vanishes on $\partial \Theta \cap \operatorname{int}(D)$,

(iii) the time derivative of $V$ along the solutions is positive definite in $\Theta$,

(iv) $P \in \partial \Theta$,

then the null solution is unstable.

The main goal is to find a function $V$ satisfying the above conditions. A possible choice can be suggested by the work of Rionero ( see [29] ) in which the stability/instability of the zero solution of the following non autonomous linear 
system is studied:

$$
\left\{\begin{array}{l}
\dot{x}=a(t) x+b(t) y \\
\dot{y}=c(t) x+d(t) y
\end{array}\right.
$$

The author introduced a new Liapunov Function depending on the invariants of the lineal matrix and apply the general theory of stability and instability of non autonomous systems. This has been generalized for a bidimensional Lotka-Volterra system (see [8]).

Remark 6.2. We note that the use of the Cetaev's theorem is not necessary since it only gives a sufficient condition of instability. However, it should be useful to show the instability of the boundary fixed points and then as a consequence to find a non trivial pullback attractor. This would also suggest the usefulness of the upper bound estimate of the dimension of the pullback attractor.

Remark 6.3. Again we have to remark that the use of non autonomous stability theory is not sufficient to obtain the uniform persistence of the system since it is required a complete study in order to avoid the existence of heteroclinic connections between the invariant sets of $\mathbb{R}_{+}^{3}$.

\section{Acknowledgments.}

This work has been partially supported by FEDER and the Spanish Ministerio de Economía y Competitividad project MTM2015-63723-P and the Consejería de Innovación, Ciencia y Empresa (Junta de Andalucía) under grant 2010/FQM314 and Proyecto de Excelencia P12-FQM-1492.

\section{References}

[1] M. Anguiano and T. Caraballo, Asymptotic behavior of a non autonomous Lorenz-84 System, Discrete and Continuous Dynamical Systems 34 39013920 (2014).

[2] V.A. Boichenko and G.A. Leonov, The Hausdorff dimension of attractors of the Lorenz system, Differentsial'nye Uravneniya, 25 1999-2000 (1989).

[3] B. Bolker, M. Holyoak, V. Krivan, L. Rowe and Schmitz O, Connecting theoretical and empirical studies of traitmediated interactions, Ecology $\mathbf{8 4}$ 1101-1114 (2003).

[4] H. Bröer, C. Simó and R. Vitolo, Bifurcations and strange attractors in the Lorenz-84 climate model with seasonal forcing, Nonlinearity 15 1205-1267 (2002).

[5] J. L. Brooks and I. D. Stanley, Predation, body size, and composition of plankton, Science, 150.3692 23-35 (1965).

[6] G. Butler, H.I. Freedman and P. Waltman, Uniformly persistent systems, Proceedings of the American Mathematical Society 96(3) 425-430 (1986). 
[7] D. Cariveau, R.E. Irwin, A.K. Brody, S.L. Garcia-Mayeya and A. Von der Ohe, Direct and indirect effects of pollinators and seed predators to selection on plant and floral traits, OIKOS 104 15-26 (2004).

[8] F. Capone, R. De Luca and S. Rionero, On the stability of non-autonomous perturbed Lotka-Volterra models, Applied Mathematics and Computation 219 (12) 6868-6881 (2013).

[9] M.F. Caruselaa, F.R. Momoa and L. Romanelli, Competition, predation and coexistence in a three trophic system, Ecological Modeling 2202349 2352 (2009).

[10] V. V. Chepyzhov and M. I. Vishik, Attractors for equations of mathematical physics, American Mathematical Society Colloquium Publications 49 (2012).

[11] R. Colucci, Coexistence in a One-Predator, Two-Prey system with Indirect Effects. Journal of Applied Mathematics, 2013, Article ID 625391, 13 pages http://dx.doi.org/10.1155/2013/625391

[12] R. Colucci, and D. Nunez, Periodic Orbits for a Three-Dimensional Biological Differential Systems. Abstract and Applied Analysis, 2013, Article ID 465183, 10 pages, http://dx.doi.org/10.1155/2013/465183

[13] J. Estes, K. Crooks, R. Holt, Ecological Role of Predators. Enciclopedia of Biodiversity, Volume 4. Academic Press, 2001

[14] Indirect effects affects ecosystem dynamics. http://www.ictp-saifr.org, 2011.

[15] J. M. Ginoux, B. Rossetto, J. L. Jamet, Chaos in a three dimensional Volterra-Gause model of predator prey type. International Journal of Bifurcation and Chaos, Vol. 15, N. 4 (2005).

[16] A. Klebanoff, A. Hastings, Chaos in One-Predator, Two-Prey Models: General Results from Bifurcation Theory. Mathematical Biosciences 122, 221233 (1994).

[17] Y. A. Kuznetsov, Elements of applied bifurcation theory, Springer, 1998.

[18] D. O. Hessen, T. Andersen, P. Brettum, B. A. Faafeng, Phytoplankton contribution to sestonic mass and elemental ratios in lakes: Implications for zooplankton nutrition. Limnology and Oceanography 48(3), 2003.

[19] J. Hulsman, F. J. Weissing, Biodiversity of plankton by species oscillations and chaos. Nature 402, 1999.

[20] P.E. Kloeden and M. Rasmussen, Nonautonomous Dynamical Systems, American Mathematical Society Providence, RI, 2011. 
[21] W. Leonard and R. May, Nonlinear aspects of competition between species. SIAM J. Appl. Math. 29, 243-275, 1975.

[22] G.A. Leonov and V.A. Boichenko, Lyapunov's direct method in the estimation of the Hausdorf dimension of attractors. Acta Appl. Math., 26, No. 1 (1992), 1-60.

[23] G.A. Leonov, Formulas for the Lyapunov dimension of Henon and Lorenz attractors. Algebra Anal., 13, No. 3 (2001), 155-170.

[24] G.A. Leonov, V. Reitmann and A.S. Slepukhin, Upper estimates for the Hausdorf dimension of negatively invariant sets of local cocycles, Doklady Mathematics, 84, No. 1 (2011), 551-554.

[25] V. Lundgren, E. Granéli, Grazer-induced defense in Phaeocystis globosa (Prymnesiophyceae): Influence of different nutrient conditions. Limnology and Oceanography, 55(5), 2010.

[26] R. Margalef, Life forms of Phytoplanktos as survival alternative in an unstable environment. Oceanologica acta, Volume 134, 1978.

[27] B. A. Menge, Indirect effects in marine rocky intertidal interaction webs: patterns and importance. Ecological Monographs 65, 1995.

[28] D. R. Merkin Introduction to the theory of stability. Springer texts in Applied Mathematics 24 (1997).

[29] S. Rionero, Stability-Instability criteria for non-autonomous systems. Atti Accad. Naz. Lincei Cl. Sci. Fis. Mat. Natur. Rend. Lincei, s.9, Mat. Appl. 20, n. 4, (2009) 347-367.

[30] O. Sarnelle, Daphnia as keystone predators: effects on phytoplankton diversity and grazing resistance. Journal of Plankton Research, Volume 27, n. $12,2005$.

[31] W. E. Snyder, A. R. Ives, Generalist predators disprut biological control by a specialist parasitoid. Ecology, 82(3), 2001.

[32] S. M. Shahruz, D. A. Kalkin, Limit cycle behavior in three or higher dimensional non linear systems: The Lotka-Volterra example. Journal of sound and vibration 246(2), 2001.

[33] H. L. Smith, H. R. Thieme, Dynamical Systems and Population Persistence. American mathematical society, 2011.

[34] J. Vandermeer, Seasonal Isochronic Forcing of Lotka Volterra Equations. Progress of Theoretical Physics, Volume 96 (1),July 1, 1996.

[35] P. Van Den Driessche, M. L. Zeeman, Three dimensional competitive LotkaVolterra Systems with no periodic orbits. Siam J. Appl. Math. Vol 58 (1), 1998. 
[36] J. Vandermeer, L. Stone, B. Blasius, Categories of chaos and fractal basin boundaries in forced predator?prey models. Chaos, Solitons \& Fractals, Volume12, Number 2 Pages 265 - 276.

[37] M. R. Walsh, D. N. Reznick, Interactions between the direct and indirect effects of predators determine life history evolution in a killifish. Pnas, www.pnas.org/cgi/doi/10.1073/pnas.0710051105.

[38] H. Wolkowicz, G. P. H. Styan, Bounds for eigenvalues using traces. Linear Algebra Appl. 29, 471?506, (1980).

[39] J. T. Wootton, Indirect Effects, Prey Susceptility, and Habitat Selection: Impacts of Birds on Limpets and Algae. Ecology, Vol.73, N.3, 1992 